\title{
Los finales en la literatura infantil contemporánea
}

\author{
Karla Fernández de Gamboa Vázquez \\ Universitat Autònoma de Barcelona
}

(Texto recibido 19 de marzo de 2018; aceptado 27 de abril de 2018; versión final 09 de mayo de 2018)

DOI: https://doi.org/10.5565/rev/jt13.756

Resumen: Las últimas décadas del siglo XX trajeron consigo una innovación notable en la literatura infantil de las sociedades postindustriales occidentales. Este cambio, tanto literario como educativo, se ha manifestado en la producción de libros con nuevos contenidos, nuevas técnicas artísticas y formas discursivas (Silva-Díaz, 2005). Entre dichas innovaciones el uso de desenlaces que distan del arquetípico final feliz ha sido una de las más polémicas en el campo de su valoración. Tomando este debate como punto de partida, el presente estudio analiza los finales de las obras de la literatura infantil contemporánea más recomendadas por la crítica especializada dirigidas a lectores de entre 8 y 10 años. Los resultados obtenidos muestran que la diversificación de los tipos de desenlace ha aumentado en los últimos años y que su uso se ha renovado adhiriéndose a las nuevas características que presenta la literatura infantil en estas primeras décadas del siglo XXI.

Palabras clave: Literatura infantil, desenlace, final

Resum: Les últimes dècades del segle XX van portar amb si una innovació notable en la literatura infantil de les societats postindustrials occidentals. Aquest canvi, tant literari com educatiu, s'ha manifestat en la producció de llibres amb nous continguts, noves tècniques artístiques i formes discursives (Silva-Díaz, 2005). Entre aquestes innovacions l'ús de desenllaços que disten de l'arquetípic final feliç ha estat una de les més polèmiques en el camp de la seva valoració. Prenent aquest debat com a punt de partida, el present estudi analitza els finals de les obres de la literatura infantil contemporània més recomanades per la crítica especialitzada dirigides a lectors d'entre 8 i 10 anys. Els resultats obtinguts mostren que la diversificació dels tipus de desenllaç ha augmentat en els últims anys i que el seu ús s'ha renovat adherint-se a les noves característiques que presenta la literatura infantil en aquestes primeres dècades del segle XXI.

Paraules clau: Literatura infantil, desenllaç, final

\begin{abstract}
The late 20th century brought a notable innovation in children's literature in Western post-industrial societies. This change, both literary and educational, has been manifested in the production of books with new content, new artistic techniques and new discursive forms (Silva-Díaz, 2005). Amongst these innovations, the use of endings which are removed from the archetypical happy ending has been one of the most controversial in the field of their assessment. Taking this debate as a point of departure, this study analyses the endings of works of contemporary children's literature aimed at readers aged 8 to 10 years, which specialized critics have recommended the most. The results obtained demonstrate that the diversification of the varieties of denouement has increased in recent years. Moreover, its use has been
\end{abstract}


renewed by complying with the new characteristics of children's literature in these first decades of the 21 st century.

Key words: Children's literature, denouement, closure, ending

\section{Introducción: la importancia de un final}

Son muchos los autores que han intentado precisar el concepto de final narrativo; no obstante, esta labor de concreción ha dado pie a diferentes teorías sobre la verdadera naturaleza que se esconde detrás del desenlace de una narración, llegando a lo que algunos consideran un callejón sin salida.

J. H. Miller (1978) consideró el final narrativo como algo “inherentemente indecidible" (1978, p. 3) haciéndose eco de la discrepancia existente entre algunos críticos sobre si las obras presentan un final o, de lo contrario, siempre permanecen "abiertas". Mientras que para unos toda narración comienza y acaba in medias res, debido a que cualquier novela aparentemente acabada puede ser reabierta, para otros, el final de una narración reside en su resolución o desenlace, hecho que proyecta una unión retrospectiva sobre la totalidad de la obra.

Estas discrepancias mencionadas por J. H. Miller (1978) nacen, precisamente, de la imposibilidad de decretar cuándo una narración está completa, pues si el final es considerado como el último lazo de un nudo, este siempre podría ser desatado, desenredado o explicado de nuevo por el narrador o por futuros acontecimientos.

En un estudio publicado pocos años después, D. A. Miller (1981), a diferencia de J. H. Miller (1978) quien usaba indistintamente ambas palabras, sustituye "final" [ending] por "cierre" [closure], partiendo del término propuesto por Smith $(1968)^{\mathrm{ii}}$, ya que sostiene que este concepto es más efectivo a la hora de hacer referencia a "las funciones de un final: para justificar el cese de la narrativa y para complementar el significado de lo que ha sido antes" (D. A. Miller, 1981, p. xi).

La diferencia entre ambos términos ha salido a colación en diversos estudios como los de Torgovnick (1981) o Segal (2007). Segal (2007), por ejemplo, coincide plenamente con Smith (1968), mientras que Torgovnick (1981) denomina "cierre" al proceso a través del cual una novela alcanza un adecuado y apropiado desenlace en relación con el inicio y desarrollo de esta, pero difiere de Smith (1968) ya que, en su opinión, las novelas con un final abierto también consiguen concluir de forma eficaz. 
Sin embargo, Phelan (1989) da un paso más en esta problemática y hace una distinción entre "cierre" [closure], lo que considera como la manera en la que una narración señala su final, y "completitud" [completeness], el grado de resolución que acompaña al desenlace. Cadden (2012), en su estudio sobre los epílogos en las novelas fantásticas infantiles, aclara los conceptos de "cierre" y "finalización" [completion] de la siguiente manera: "El cierre tiene que ver con el mecanismo de la progresión narrativa (...) mientras que la finalización hace referencia a las "inestabilidades" que impulsan la progresión y dirigen los intereses de los lectores implícitos (...)” (p. 344).

Sea como fuere, más allá del debate sobre la precisión de estos términos, todos los autores coinciden con lo que Kermode (1966) denominó "el sentido de un final”, es decir, la importancia del final como un elemento organizador, no solo de la propia trama de la obra, sino también para dar sentido al mundo que nos rodea. Así lo apuntaba igualmente Torgovnick (1981), para quien la apreciación de los desenlaces es una forma de evaluar y organizar la experiencia personal:

En parte, valoramos los finales porque el patrón retrospectivo usado para dar sentido a los textos corresponde a un proceso que se utiliza para dar sentido a la vida: el proceso de mirar hacia atrás sobre los acontecimientos e interpretarlos a la luz del "cómo salieron las cosas". (p. 5).

También en el campo específico de la literatura infantil y juvenil se ha reflejado el interés por la tendencia a la búsqueda de patrones de la mente humana:

Como seres humanos, dotados de pensamiento narrativo, buscamos darle un cierre a lo que nos acontece, por lo que vamos ordenando la experiencia personal en episodios y etapas. La manera en que anticipamos el final afecta a cómo leemos el presente: nos permitimos añadir un significado anticipado a nuestro "ahora", asumiendo que el esperado "entonces" le dará sentido a la trama. (...) En la creación de tales estructuras narrativas vitales esperamos que exista coherencia entre el comienzo, el desarrollo y el final, ya que, casi siempre, los dos primeros elementos son reorganizados en función del último. (Bellorín y Silva-Díaz, 2010, p. 129).

Por lo tanto, no es de extrañar que el desenlace sea un componente fundamental en las narraciones, no solo por su innegable importancia como elemento constructivo, sino también por la intención didáctica que se esconde en el tipo de final utilizado en cada relato, especialmente en el contexto de la literatura infantil. Tal y como subraya Colomer (1996), los estudios de la literatura infantil siempre han considerado el final de la obra como un elemento 
esencial para el mensaje educativo que desee transmitirse, ya que el lector podría inferir normas de conducta en base al triunfo o a la derrota del protagonista.

\section{Cuántos libros, tantos finales, pero ¿todos cuentan?}

Hasta la década de los años 70 no cabía duda de que en los cuentos infantiles, con la salvedad de algunos cuentos tradicionales, por muchas penurias que los protagonistas tuviesen que pasar, todo acabaría bien.

Por mucho que el lector se hubiera horrorizado, llorado o estado en tensión, podía tener la seguridad de que al final de la lectura experimentaría un alivio definitivo. El conflicto desaparecería para siempre y el lector podría emerger de su viaje literario con la satisfacción de la felicidad obtenida. (Colomer, 2005, p. 207).

Por lo que no es de extrañar, como señala Sánchez García (2011), que en la tradición española la fórmula de cierre más habitual y extendida haya sido vivieron felices y comieron perdices, empleada "para dejar claro que, a pesar de todos los avatares descritos, los protagonistas tendrán un final feliz, o sea, una vida mucho más placentera que la narrada, felicidad que festejan para compensar el hambre imperante comiendo perdices" (p. 16).

Sin embargo, como ya reveló el estudio realizado por Colomer (1998), la literatura infantil y juvenil moderna, a través del uso de diferentes tipos de finales, ha ido ampliando las posibilidades narrativas, pues solo el $60 \%$ de las obras infantiles de las dos últimas décadas del siglo pasado continuaban la tradición de resolver el conflicto inicial haciéndolo desaparecer, es decir, los finales ya no eran siempre felices.

Junto con el uso de los nuevos desenlaces, no se hizo esperar la polémica sobre si son o no adecuados moralmente, de si pueden ser entendidos o asimilados psicológicamente por los niños, o de si les ayudará a crecer como lectores (Colomer, 2005), siendo el final negativo el más cuestionado de todos.

El psicoanálisis, y sobre todo Bettelheim (1975), valoró tan positivamente la virtud tranquilizadora de los finales felices que dentro de esta corriente crítica se rechazaron totalmente los desenlaces de la literatura infantil que no lo fuesen.

Muchos de estos cuentos nuevos tienen desenlaces tristes, que no consiguen proporcionar la huida y el alivio necesarios, después de los hechos temibles que se han producido a lo largo del cuento, para que el niño disponga de la fuerza suficiente al enfrentarse a sus desventuras. Si carece de estos finales alentadores, el niño, después 
de oír el relato, sentirá que no existe esperanza alguna de solucionar sus problemas. (Bettelheim, 2010, p. 199).

Es por ello por lo que la discusión sobre la idoneidad de los diferentes finales de las obras de la literatura infantil ha apuntado principalmente a la supremacía del final feliz, y son pocos los estudios que analizan otros posibles finales ${ }^{\text {iii }}$. Para Nikolajeva (2005, p. 104), por ejemplo, “el final feliz es uno de los criterios más importantes en las definiciones convencionales de la literatura infantil, así como uno de los prejuicios más comunes al respecto”.

Además, llama la atención que en la mayoría de las investigaciones que analizan las preferencias de los lectores por un tipo de final u otro en contextos muy dispares (Goldman y Varnhagen, 1983; León y Marchesi, 1987; Goldman y Kantor, 1993) el final triste se presenta como la única alternativa al arquetípico vivieron felices y comieron perdices. No obstante, partiendo de la tipología propuesta por los estudios de Colomer (ver Colomer 1996, 1998, 2005, 2010, 2015 y 2016), los desenlaces de las obras de literatura infantil y juvenil podrían clasificarse del siguiente modo:

- Positivo por desaparición del problema: en el que el problema planteado al comienzo de la historia se resuelve satisfactoriamente.

- Positivo por asunción del problema: es una nueva versión del final feliz, donde lo positivo radica en la aceptación del conflicto, ya que este no se resuelve, sino que los personajes aprenden a vivir con él.

- Abierto: que permite al lector varias interpretaciones, puesto que se deja en el aire la incógnita sobre cómo el protagonista podría actuar en relación con el problema planteado $^{\text {iv }}$.

- Negativo: en el que el conflicto planteado al inicio de la narración no se resuelve satisfactoriamente para el protagonista, ya que este no consigue su objetivo o no existe un proceso de mejoramiento para él ${ }^{\mathrm{v}}$.

- Mezclado: cuando en una misma narración se mezclan elementos de más de un tipo de final.

A continuación, pasaremos a analizar los finales de un conjunto de obras contemporáneas dirigidas a lectores entre 8 y 10 años con el objetivo no solo de conocer su tipología y características, sino también de explorar cuáles son las tendencias imperantes en cuanto a los desenlaces narrativos de la literatura infantil actual. 


\section{Selección de las obras analizadas}

En el presente apartado expondremos los criterios de selección del conjunto de obras que han compuesto el corpus de análisis de este estudio. Se han adoptado por continuidad, y en la medida de lo posible, los mismos criterios de elección adoptados por Colomer (1998) tanto respecto a las características internas de las obras como a las fuentes de selección. Se ha decidido tomar como referencia la metodología seguida por Colomer (1998) porque su estudio, tal y como acabamos de mencionar, además de sentar las bases de la clasificación de los desenlaces en las narrativas infantiles, fue la primera investigación desarrollada en el marco estatal con el objetivo de caracterizar la literatura infantil y juvenil publicada en España desde finales de los años setenta hasta la década de los noventa.

El primer criterio de selección se refiere a la calidad de las obras, puesto que las narraciones consideradas de calidad "son las que mejor pueden evidenciar los presupuestos educativos y literarios que configuran la literatura infantil y juvenil”' (Colomer, 1998, p. 148). Es por ello por lo que se han tenido en cuenta exclusivamente aquellas obras recomendadas por la crítica especializada, ya que dicho corpus "puede ser considerado hoy en día, y a falta de otros indicadores, la "mejor narrativa" actual" (Colomer, 1998, p. 149).

Para ello, se ha revisado la crítica especializada actual, un proceso que ha partido de la valoración de la veintena de fuentes de selección que componen la base de datos relacional desarrollada por el grupo GRETEL (Grupo de Investigación de literatura infantil y juvenil y educación literaria de la Universitat Autònoma de Barcelona). Tras dicho análisis, se han incluido aquellas que mejor se ajustan a las principales premisas seguidas por Colomer (1998):

Nuestro trabajo requería selecciones bibliográficas que cubrieran el período escogido y que no distinguieran entre producción autóctona y traducción. Cualquier estudio debe situarse en un contexto determinado y por ello la óptica escogida hace referencia a la valoración de las obras desde la perspectiva de Cataluña, lo cual nos ha llevado a priorizar las selecciones de mayor influencia en este ámbito. También era necesario que las selecciones especificaran la edad de los destinatarios si se quería proceder a un análisis evolutivo de los requerimientos literarios implicados. (p. 151).

En base a ello, las fuentes de selección consideradas en el presente estudio han sido un total de dieciséis, entre las que se encuentran selecciones bibliográficas, premios y revistas especializadas en literatura infantil y juvenil de ámbito tanto autonómico y estatal como internacional $^{\mathrm{vi}}$. 
El segundo criterio de selección del presente estudio es el referido a la delimitación temporal y lingüística de las obras. El criterio de "contemporaneidad" comprende aquellas obras que han sido publicadas durante el período 2003-2013. Además, el análisis contempla las obras publicadas en castellano o catalán con independencia de su lengua original, por ser estos los idiomas dominados por el público infantil del contexto en el que se desarrolla el presente estudio. Sin embargo, quedan excluidas de este criterio aquellas obras reeditadas, a excepción de aquellas obras traducidas al castellano o al catalán por primera vez en este período en vista de que, a pesar de haber sido publicadas antes de estas fechas en su lengua original, forman parte del conjunto de lecturas de calidad ofrecido a las niñas y niños en este período.

En tercer lugar, el criterio respecto a las características internas de las obras es el que probablemente más ha variado comparado con la investigación realizada por Colomer (1998). Por un lado, se han seguido las principales directrices de escoger obras literarias y de narrativa moderna, por lo que se han desestimado tanto las obras de poesía, las teatrales y las consideradas "no-fícción" o de conocimientos, así como los cuentos populares, las leyendas, las narraciones mitológicas, etc.

Por otro lado, se ha acotado la franja de edad de los lectores a las que se dirigen las obras. Mientras que el análisis de Colomer (1998) incluye obras recomendadas para lectores desde los 5 hasta los 15 años, la actual investigación se centra en las dirigidas a lectores entre 8 y 10 años. Esta delimitación del corpus se debe principalmente al interés por observar cuáles son las competencias en el campo literario que estas narraciones presuponen de su lector implícito que en estas edades ya es considerado un lector autónomo.

Además, a diferencia del estudio de Colomer (1998) y debido a la proliferación de este tipo de obras, también se han tenido en cuenta aquellas obras con una clara preponderancia de la ilustración a efectos de construcción del significado, como es el caso del álbum, exceptuando otro tipo de obras que también emplean un lenguaje visual como lo son las novelas gráficas y los cómics.

Por lo tanto, en base a estos criterios, el análisis se refiere a narraciones literarias actuales para niñas y niños entre los 8 y los 10 años. A partir de las dieciséis fuentes de selección y siguiendo los criterios apenas descritos surgió un primer corpus de aproximadamente 1.500 obras. Siendo esta una cantidad inabarcable para el análisis previsto, se optó por escoger aquellas obras que hubieran sido citadas en las selecciones o hubiesen obtenidos premios en cinco ocasiones o más. De este modo, la selección definitiva consta de 36 obras (el listado de las obras seleccionadas puede consultarse en el anexo). 


\section{Los finales de la literatura infantil contemporánea}

A pesar de que el final feliz ha sido aparentemente una de las constantes de la literatura infantil y juvenil, solo la mitad de las narraciones analizadas presentan dicho final. Entre aquellas obras que deciden alejarse del final positivo tradicional, el final abierto parece ser la opción predilecta, pues una cuarta parte del total de obras analizadas acaban de este modo. Además, aunque en menor medida, también se hallan finales positivos por asunción del problema $(11,11 \%)$, negativos $(8,33 \%)$ y mezclados $(5,56 \%)$. Estos últimos, en cambio, se reducen a dos obras que están compuestas por varios relatos que acaban de forma diversa, por lo que realmente no existe ningún caso entre las obras analizadas que presente una mezcla de elementos de uno u otro de los tipos de desenlace en una misma narración.

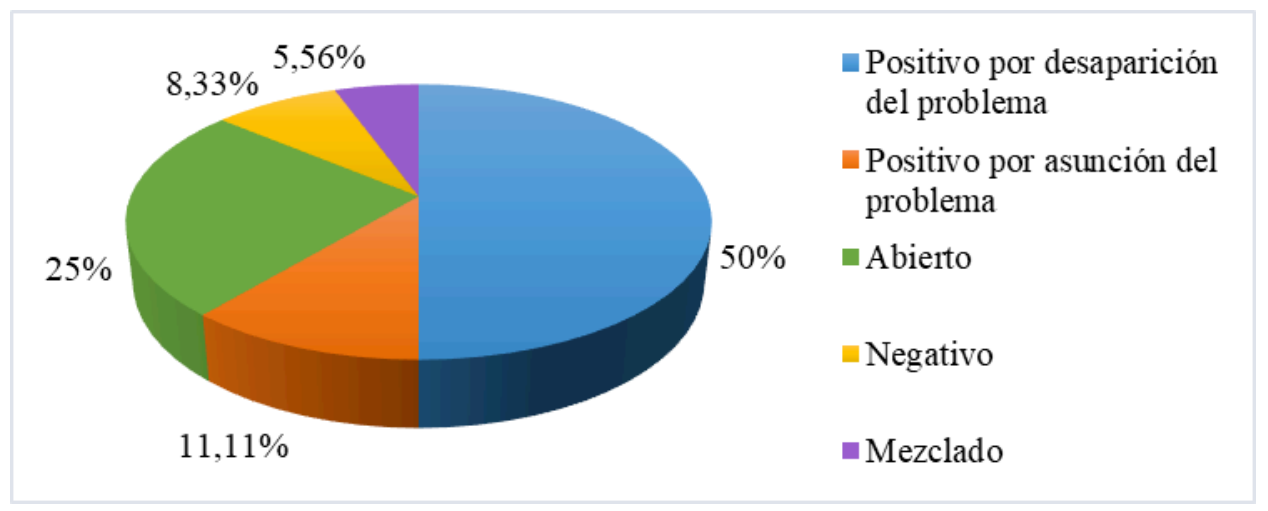

Figura 1: Tipos de desenlace.

El final positivo por desaparición del problema se emplea principalmente en las obras que adoptan modelos de la literatura tradicional y en las de fantasía moderna cuyo conflicto es externo y desaparece. En la mayoría de este tipo de obras, tal y como ocurre también en la tradición de estos géneros literarios, el conflicto se resuelve gracias al ingenio y la valentía de los protagonistas que se imponen a las malas intenciones de los antagonistas. No obstante, también se dan casos, aunque en menor medida, en los que el conflicto se resuelve o bien por la impericia de los antagonistas, como en La leyenda de la serpiente (Anderson y Ibatoulline, 2006) donde los balleneros nunca llegan a capturar a la serpiente marina; o bien por la conducta de los protagonistas en ausencia de adversarios a los que derrotar. Es más, en algunas de estas obras, los cambios de carácter de los protagonistas, en los que siempre reside una enseñanza moral, son imprescindibles para la resolución del conflicto externo, pues cuando los protagonistas rechazan actitudes racistas, egoístas y orgullosas es cuando encuentran la forma de solucionar el problema. Más aún, algunas veces, como en Pájaros en 
la cabeza (Rosell y Torrão, 2004), son los mismos antagonistas los que cambian de actitud como resultado del escarmiento que les da el protagonista. Ello nos habla de la característica moderna de una caracterización más psicológica y personalizada de protagonistas y antagonistas derivados de la literatura tradicional y enlaza con la adscripción al final feliz de las pocas obras en las que la trama gira en torno a un conflicto interno del protagonista que concluye con su maduración personal, como en Tres hermanas ladronas (Arnal y Odriozola, 2007).

El uso del desenlace positivo por asunción del problema ya era un recurso común en las obras infantiles de finales del siglo XX que lidiaban con la complejidad de los sentimientos humanos, y ahora se continúa empleando especialmente en narraciones que tratan temas como la aceptación de uno mismo o la muerte de un ser querido. No obstante, actualmente esta nueva versión del final feliz parece renovarse por su uso en tramas que también abordan una problemática social, como ¡Vamos a ver a papá! (Schimel y Ribera, 2010) o Habría que... (Lenain y Tallec, 2005) que versan sobre diferentes problemas de la sociedad actual, tales como la emigración, las guerras, el hambre y la miseria, el abuso de poder o la responsabilidad medioambiental. Todas estas cuestiones se abordan desde un punto de vista infantil y se presentan como asuntos complejos que no disponen de una solución simple o inmediata que haga desaparecer por completo el conflicto planteado ni las dificultades que genera a los protagonistas. De modo que desembocan simplemente en la comprensión del problema y en una mejoría de la situación que se mostraba al inicio de la narración. Debido a la dificultad de estos aspectos, los finales de ambas obras también comparten cierto grado de apertura pues, a pesar del eco positivo de ambos, el lector no sabe a ciencia cierta qué ocurrirá después o cómo se desarrollarán los acontecimientos.

El final abierto está presente en obras que diluyen la consistencia narrativa y que podrían dividirse en tres principales grupos. En primer lugar, aparece en aquellas obras que se proponen abrir la puerta al debate sobre cuestiones sociales tan diversas como el abandono y maltrato animal en Un día un perro (Vincent, 2004) o la apatía y la alienación causados por el pragmatismo y la burocracia en La cosa perdida (Tan, 2005). En segundo lugar, este desenlace también es utilizado por obras que relatan la vida y evolución interna de los personajes, como ocurre, por ejemplo, en Shola y Angeliño (Atxaga y Valverde, 2006), por lo que el conflicto inicial pasa a un segundo plano en aras de la maduración y cambio personal de los protagonistas. En estos casos, el problema planteado al comienzo no es realmente el conflicto narrativo, sino el desencadenante de las acciones que darán pie a la transformación y crecimiento de los personajes a través de la interacción entre ellos. En tercer lugar, cuando el 
debilitamiento del conflicto como núcleo narrativo $\mathrm{y}$, por tanto, de la estructura narrativa tradicional es aún mayor, el uso del final abierto puede derivar en un juego metaliterario y humorístico como ocurre, por ejemplo, en el cuento de La tortuga y el pelo de liebre inscrito en El apestoso hombre queso y otros cuentos maravillosamente estúpidos (Scieszka y Smith, 2004), en el que se parodian varios cuentos tradicionales. De este modo, estas obras con características propias de la literatura posmoderna parecen presuponer un lector implícito con cierta competencia literaria, aunque para facilitar la identificación, comprensión y fruición de estas licencias literarias se amparan en la popularidad de los cuentos clásicos, referentes comunes y conocidos por los lectores de estas edades.

Asimismo, cabe señalar que, de los diferentes tipos de finales narrativos, el abierto es el que más directamente apela a la competencia interpretativa del lector. Una apelación que se ve acrecentada, aún más, por la función narrativa de las ilustraciones de los álbumes con finales abiertos, ya que la combinación del código textual y visual resulta clave en algunos de estos álbumes, como en Nublado con probabilidades de albóndigas (Barrett y Barrett, 2012) o en En Matadegolla (Duran y Danide, 2010), donde el lector acaba inmerso en un juego literario por intentar averiguar si la historia podría volver a repetirse; o, como por ejemplo, en Zorro (Wild y Brooks, 2005), donde de las ilustraciones de las últimas dobles páginas se desprende una posible resolución esperanzadora, algo requerido para suavizar la carga negativa de los acontecimientos previos.

Por último, el final negativo con intención aleccionadora resulta algo común en obras propias de géneros de la literatura tradicional como las fábulas y se perpetúa actualmente en aquellas obras que adoptan dichos modelos, como en El príncipe de los enredos (Aliaga y Olmos, 2009) o en El ratón del señor Maxwell (Asch y Asch, 2004). En ambos títulos los personajes acaban pagando caros sus errores, si bien la reacción que suscitan en el lector es opuesta: mientras que el final de El príncipe de los enredos (Aliaga y Olmos, 2009) produce un impacto emocional que invita a la reflexión de un modo más tradicional, las últimas escenas de El ratón del señor Maxwell (Asch y Asch, 2004), propias de cualquier gag humorístico, sitúan al lector en una posición de superioridad sobre el personaje que recibe el escarmiento. Este último título es un ejemplo de la tendencia de este tipo de desenlaces a enmascarar el mensaje moralizante entre carcajadas, hasta el punto de desvirtuar casi por completo cualquier posible lección ética. El uso del final negativo sin que suponga una sanción moral se convierte así en una de las estrategias subversivas propias de la literatura infantil posmoderna, especialmente utilizada para parodiar los finales felices de los cuentos clásicos. La hallamos, por ejemplo, en la hiperbolización de Lola se embala y otros cuentos 
terribles (Bredow y Kuhl, 2013), una obra adscrita a la línea clásica de Pedro Melenas (Hoffmann, 1987), en algunos de los cuentos del ya aludido El apestoso hombre queso y otros cuentos maravillosamente estúpidos (Scieszka y Smith, 2004) o en El contador de cuentos (Saki y Rivera, 2008), donde la crítica al didactismo y la moralidad de la literatura infantil es el eje principal de la narración y el poder subversivo del final negativo llega incluso a explicitarse a través de la discusión de los personajes.

\section{La función de los finales en la formación del lector}

Considerando que la mitad de las obras analizadas poseen un desenlace que dista del positivo tradicional, podría creerse que la desconfianza que ha generado el uso de diferentes tipos de final se está disipando, especialmente en el ámbito de la crítica especializada de literatura infantil. Ahora bien, algunos mediadores parecen seguir cuestionándose la idoneidad de incluir narraciones con multiplicidad de finales en las bibliotecas escolares o de aula, a pesar de que esta diversidad resulta esencial para la formación literaria de los lectores.

Es sabido que la literatura infantil, adaptándose al contexto en el que se produce, cumple tres funciones principales: el aprendizaje del lenguaje y de las formas literarias, el acceso al imaginario colectivo y la socialización cultural (Colomer, 2005 y 2010). Por consiguiente, también lo hacen los desenlaces de las obras, tal y como se ha descrito en el anterior apartado. Más aún, para garantizar que dichas funciones se cumplan y, que el lector implícito pueda desarrollar su competencia literaria es necesario que disponga de un corpus heterogéneo de obras de calidad que ofrezca múltiples experiencias literarias.

Como se muestra en el presente estudio, las narraciones que traicionan las expectativas de los lectores de un final feliz aportan una experiencia literaria distinta a aquellas que poseen un desenlace tradicional. Bellorín y Silva-Díaz (2010) señalan que los finales sorprendentes, aquellos que no cumplen las expectativas del lector, "pueden permitir a los niños explorar la manera en que funcionan los artefactos narrativos y pensar acerca de lo que les puede ofrecer la literatura" (p. 132). De modo que, pese a la polémica suscitada en torno a los nuevos desenlaces, estas propuestas resultan esenciales en el aprendizaje de literatura, especialmente para los lectores en formación. Razón por la cual es fundamental que los mediadores les brindemos la posibilidad de acceder a este tipo de narraciones. 


\section{Conclusiones}

Los resultados obtenidos indican que la mitad de las obras analizadas se alejan del tradicional final feliz. Es más, si se comparan los datos del presente estudio con la investigación realizada por Colomer (1998), se constata que las obras contemporáneas dirigidas a lectores de entre 8 y 10 años con un final que no sea el positivo por desaparición del conflicto aumentan 29,1 puntos porcentuales. Un incremento motivado especialmente por el aumento del final abierto (14,55 puntos porcentuales) y también, aunque en menor medida, del negativo (6,83 puntos porcentuales) y del positivo por asunción del problema (2,16 puntos porcentuales). Por lo tanto, la tendencia a la diversificación de los tipos de desenlace que ya destacaba en la literatura infantil de finales del siglo XX es incluso más acusada entre las obras actuales.

Asimismo, los desenlaces de la literatura infantil actual no solo presentan una mayor diversidad, sino que también se renuevan adhiriéndose a las nuevas características que presenta la literatura infantil en estas primeras décadas del siglo XXI, entre las que destacan: una caracterización más psicológica y personalizada de protagonistas y antagonistas, especialmente de aquellos derivados de la literatura tradicional; el aumento de temas que abordan una problemática social; y la apuesta por obras con rasgos propios de la literatura posmoderna, como lo son la metaficción y la parodia como estrategia subversiva.

Por otro lado, la proliferación del álbum en la producción actual ha derivado en un considerable aumento del valor discursivo de las ilustraciones, ya que resultan imprescindibles para la comprensión global de la narración. En estos casos, las ilustraciones permiten introducir mayor complejidad literaria al mismo tiempo que posibilitan al lector en formación afrontar el reto que puede suponer la lectura y desarrollar, por consiguiente, su competencia literaria.

Por último, el acceso a obras con diferentes tipos de final resulta esencial para la formación literaria del lector, dado que cada tipología moviliza aprendizajes específicos y aporta experiencias literarias diversas.

\section{Referencias}

Bellorín, B., y Silva-Díaz, C. (2010). Los finales sorprendentes en los libros-álbum. En T. Colomer, B. Kümmerling-Meibauer y C. Silva-Díaz (Eds.), Cruce de miradas: Nuevas aproximaciones al libro-álbum (pp. 128-141). Barcelona: Banco del Libro-GRETEL.

Bettelheim, B. (1975). The uses of enchantment. The meaning and importance of fairy tales. Londres: Thames and Hudson. 
Bettelheim, B. (2010). Psicoanálisis de los cuentos de hadas. Barcelona: Crítica.

Cadden, M. (2012). All Is Well: The Epilogue in Children's Fantasy Fiction. Narrative, 20(3), 343-356. DOI: https//doi.org/10.1353/nar.2012.0018

Colomer, T. (1996). ¿Cómo terminan los cuentos? Espacios para la Lectura, 2, 6-7.

Colomer, T. (1998). La formación del lector literario: narrativa infantil y juvenil actual. Madrid: Fundación Germán Sánchez Ruipérez.

Colomer, T. (2005). El desenlace de los cuentos como ejemplo de las funciones de la literatura infantil y juvenil. Revista de Educación, Número extraordinario, 203-216. Recuperado de http://www.revistaeducacion.mec.es/re2005/re2005 16.pdf

Colomer, T. (2010). Introducción a la literatura infantil y juvenil actual. Madrid: Síntesis.

Colomer, T. (2015). The construction of meaning through endings: Different types of endings in children's stories. Conferencia presentada en la $25^{\text {th }}$ EECERA Annual Conference: Innovation, Experimentation and Adventure in Early Childhood, Barcelona, 8 septiembre. Recuperado de https://www.eecera.org/wpcontent/uploads/2015/09/Teresa-Colomer-EECERA-2015-Keynote-Presentation.pdf

Colomer, T. (2016). ¿Fueron felices y comieron perdices? CLIJ: Cuadernos de literatura infantil y juvenil, 269, 6-14.

Goldman, S. R., y Kantor, R. J. (1993). The limits of poetic license: When shouldn't an ending be happy? Poetics, 22, 135-150. DOI: https://doi.org/10.1016/0304$\underline{422 X(93) 90025-\mathrm{C}}$

Goldman, S. R., y Varnhagen, C.K. (1983). Comprehension of Stories with No-obstacle and Obstacle Endings. Child Development 54, 980-992. doi:10.2307/1129902

Hoffmann, H. (1987). Pedro Melenas: Historias muy divertidas y estampas aún más graciosas. Palma de Mallorca: José J. de Olañeta.

Kermode, F. (1966). The sense of an ending: Studies in the theory of fiction. Oxford: Oxford University Press.

León, J. A., y Marchesi, A. (1987). La influencia de variables cognitivas en el recuerdo del cuento y su valoración en la función de la edad. Infancia y aprendizaje, 37, 19-31. DOI: https://doi.org/10.1080/02103702.1987.10822145

Miller, D. A. (1981). Narrative and its discontents: Problems of closure in the traditional novel. Princeton: Princeton University Press.

Miller, J. H. (1978). The problematic of ending in narrative. Nineteenth-century Fiction, 33(1), 3-7. DOI: http://doi.org/10.2307/2932923 
Nikolajeva, M. (2005). Aesthetic approaches to children's literature: An introduction. Lanham, Maryland: The Scarecrow Press.

Phelan, J. (1989). Reading people, reading plots: Character, progression, and the interpretation of narrative. Chicago: University of Chicago Press.

Sánchez García, S. (2011). Relaciones intertextuales y competencia literaria en la obra narrativa de Fernando Alonso. Ocnos, 7, 7-22. DOI: http://dx.doi.org/10.18239/ocnos_2011.07.01

Segal, E. (2007). Narrativity and the closure of event sequences. Amsterdam International Electronic Journal for Cultural Narratology (AJCN), 4, s.p. Recuperado de http://cf.hum.uva.nl/narratology/a07_segal.htm

Silva-Díaz, M. C. (2005). Libros que enseñan a leer: álbumes metaficcionales y conocimiento literario (Tesis inédita de doctorado). Bellaterra: Universidad Autónoma de Barcelona. Recuperado de http://www.tdx.cat/handle/10803/4667

Smith, B. H. (1968). Poetic closure: A study of how poems end. Chicago: University of Chicago Press.

Torgovnick, M. (1981). Closure in the novel. Princeton: Princeton University Press.

\footnotetext{
${ }^{\mathrm{i}}$ Todas las traducciones al castellano de las citas bibliográficas que están escritas originalmente en inglés han sido realizadas por la autora de este artículo.

ii Smith (1968) consideraba que una estructura podía considerarse "cerrada" cuando se experimentaba como integral siendo esta coherente, completa y estable.

iii Cabe destacar los trabajos de Colomer (véase Colomer 1996, 1998, 2005, 2010, 2015 y 2016) en los que analiza y describe los diferentes finales que proliferaron en la literatura infantil a partir de finales de la década de los 70, y el estudio sobre los finales sorprendentes en los libros-álbum de Bellorín y Silva Díaz (2010) en el que se describen los patrones de ocultamiento y revelación característicos de este tipo de finales.

${ }^{\text {iv }}$ En este tipo de desenlaces "el lector no puede saber, pues, con certeza lo que ha ocurrido en la historia narrada o lo que ocurrirá en aspectos esenciales del relato a partir del momento en el que la narración se interrumpe" (Colomer, 1998, p. 171).

${ }^{\vee}$ Este tipo de final también ha sido denominado disonante por autores como Nikolajeva (2005), debido a la discrepancia entre el desenlace estructural de la narración (el final apropiado de la trama) y el psicológico (el que equilibra los conflictos personales del protagonista).

vi Quins llibres...? del Seminario de Bibliografía Infantil y Juvenil de l'Associació de Mestres "Rosa Sensat"; The White Ravens, A Selection of International Children's and Youth Literature de la Internationale Jugendbibliothek de Munich; Libros con estrella, selección bibliográfica creada por la Fundación Germán Sánchez Ruipérez y CEGAL; Libros escogidos de literatura infantil y juvenil, de la Red de Selección de Libros Infantiles y Juveniles; $L o+$, guía de recomendaciones elaborada por el CILIJ de la Fundación Germán Sánchez Ruipérez; Històries menudes, catálogo de Abacus cooperativa; Los mejores libros para niños y jóvenes, del Banco del Libro de Venezuela; las recomendaciones realizadas por el grupo de investigación GRETEL; Premio Nacional de Literatura, modalidad literatura infantil y juvenil; Premio Lazarillo, convocado por la OEPLI; Premio Serra d'Or, modalidad de literatura infantil y juvenil; Premio de literatura Atrapallibres, convocado por ClijCAT; Faristol, Revista del Llibre Infantil i Juvenil; Peonza, Revista de Literatura Infantil y Juvenil; CLIJ, Cuadernos de literatura infantil y juvenil; Notícia de Llibres Infantils i Juvenils.
} 


\section{Anexo}

Aliaga, R., y Olmos, R. (2009). El príncipe de los enredos. Zaragoza: Edelvives.

Anderson, M. T., y Ibatoulline, B. (2006). La leyenda de la serpiente. Barcelona: Juventud.

Arboleda, D., y Sagospe, R. (2013). Prohibido leer a Lewis Carroll. Madrid: Anaya.

Arnal, T., y Odriozola, E. (2007). Tres hermanas ladronas. Pontevedra: OQO.

Asch, F., y Asch, D. (2004). El ratón del señor Maxwell. Barcelona: Juventud.

Asch, F., y Asch, D. (2007). Los ratones de la señora Marlowe. Barcelona: Juventud.

Atxaga, B.. y Valverde, M. (2006). Shola y Angeliño. Boadilla del Monte: SM.

Barrett, J., y Barrett, R. (2012). Nublado con probabilidades de albóndigas. Barcelona: Corimbo.

Blanco, R. (2006). Cuentos pulga. Barcelona: Thule.

Bredow, W. von, y Kuhl, A. (2013). Lola se embala y otros cuentos terribles. Barcelona: Takatuka.

Canal, E., y Gubianas, V. (2008). Un somni dins el mitjó. Barcelona: Animallibres.

Carballeira, P., y Hénaff, C. (2006). Smara. Sevilla: Kalandraka.

Chen, J. H. (2004). El caballo mágico de Han Gan. Barcelona: Corimbo.

Duran, T., y Danide (2010). En Matadegolla. Barberà del Vallès: Fil d'Aram.

Eudave, C., y Muñiz, J. (2010). Papá Oso. Mataró: A buen paso.

Funke, C., y Kehn, R. (2006). Cuando Papá Noel cayó del cielo. Madrid: Siruela.

Gil, C., y Castagnoli, A. (2007). El caballero Pepino. Pontevedra: OQO.

Hole, S. (2007). El final del verano. Madrid: Kókinos.

Janisch, H., y Blau, A. (2006). Mejillas rojas. Santa Marta de Tormes: Lóguez.

Jové, J. M., y Tha (2006). L’home del sac. Barcelona: La Galera; Cercle de Lectors.

Lenain, T., y Tallec, O. (2005). Habría que... Madrid: Kókinos.

Lluch, E., y Fransoy, M. (2004). De Satanasset a Aletes-de-Vellut. Barcelona: Barcanova.

Noguès, J.-C., y Romby, A. (2003). El genio del jazmín. Barcelona: Zendrera Zariquey.

Núñez, M. L., y Lima, T. (2006). Huevos duros. Pontevedra: OQO.

Ocelot, M. (2007). Azur \& Asmar. Boadilla del Monte: SM.

Pintus, É., y Saillard, R. (2011). Hambre de Lobo. Barcelona: Océano.

Rosell, J. F., y Torrão, M. (2004). Pájaros en la cabeza. Pontevedra: Kalandraka.

Saki, y Rivera, A. M. (2008). El contador de cuentos. Barcelona: Ekaré.

Schimel, L., y Rivera, A. M. (2010). ¡Vamos a ver a papá! Barcelona: Ekaré. 
Scieszka, J., y Smith, L. (2004). El apestoso hombre queso y otros cuentos maravillosamente estúpidos. Sant Adrià de Besòs: Thule.

Serres, A., y Heitz, B. (2010). Terrible. Barcelona: Libros del Zorro Rojo.

Tan, S., (2005). La cosa perdida. Cádiz: Barbara Fiore.

Turkowski, E. (2007). Estaba oscuro y sospechosamente tranquilo. Barcelona: Libros del Zorro Rojo.

Vincent, G. (2004). Un día, un perro. Barcelona: Zendrera Zariquey.

Wiesner, D. (2003). Los tres cerditos. Barcelona: Juventud.

Wild, M. y Brooks, R. (2005). Zorro. Caracas: Ekaré

Información sobre la autora:

Karla Fernández de Gamboa Vázquez es diplomada en Educación Infantil y graduada en Educación Primaria (UPV / EHU). Máster en Biblioteca Escolar y Promoción de la Lectura (UAB y UB), en Libros y Literatura Infantil y Juvenil (UAB y el Banco del Libro de Venezuela) y en Investigación en Didáctica de la Lengua y la Literatura (UAB). Trabajó como Personal Investigador en Formación en el Departamento de Didáctica de la Lengua y la Literatura, y de las Ciencias Sociales de la Universidad Autónoma de Barcelona y ha sido profesora de didáctica de la literatura infantil en la Universidad del País Vasco. Actualmente está finalizando el Doctorado en Educación en GRETEL (Grupo de Investigación de literatura infantil y juvenil y educación literaria de la UAB).

Email: karla.fernandezdegamboa@gmail.com

Para citar este artículo:

Fernández de Gamboa Vázquez, K. (2018). Los finales en la literatura infantil contemporánea. Bellaterra Journal of Teaching \& Learning Language \& Literature, 11(1), 27-42. DOI: https://doi.org/10.5565/rev/jt13.756

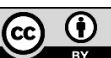

